\title{
All-fibered chalcogenide based continuous-wave parametric amplification in the mid-infrared
}

\author{
Sida Xing ${ }^{1}$, Davide Grassani ${ }^{1, *}$, Svyatoslav Kharitonov ${ }^{1}$, Camille-Sophie Brès ${ }^{1}$ \\ ${ }^{\text {I} E c o l e ~ P o l y t e c h n i q u e ~ F e ́ d e ́ r a l e ~ d e ~ L a u s a n n e, ~ P h o t o n i c ~ S y s t e m s ~ L a b o r a t o r y ~(P H O S L), ~ S T I-I E L, ~ S t a t i o n ~ 11, ~ C H-1015 ~ L a u s a n n e, ~ S w i t z e r l a n d ; ~}$ \\ *davide.grassani@epfl.ch
}

\begin{abstract}
We demonstrate parametric amplification around $2 \mu \mathrm{m}$ in a dispersion engineered tapered microstructured chalcogenide fiber. Almost $5 \mathrm{~dB}$ of signal amplification was achieved by $125 \mathrm{~mW}$ coupled power from a thulium-doped fiber pump laser.

OCIS codes: (060.4005) Microstructured fibers; (190.4410) Nonlinear optics, parametric processes; (230.4480) Optical amplifiers
\end{abstract}

\section{Introduction}

Continuous-wave $(\mathrm{CW})$ pumped parametric wavelength conversion and amplification was demonstrated to possess ultrahigh response together with reduced nonlinear noise, enabling high performances in all-optical processing using highly nonlinear fibers in the telecom band [1]. Due to the excessive loss of silica fibers beyond $2 \mu \mathrm{m}$, several new platforms were developed to translate parametric processes in the mid-infrared (MIR). Parametric amplification was achieved in planar silicon waveguide [2] and tapered $\mathrm{As}_{2} \mathrm{Se}_{3}$ fiber [3] in the $2 \mu \mathrm{m}$ wavelength range using a pulsed pump. However, both platforms have high propagation loss in orders of $\mathrm{dB} / \mathrm{cm}$, inhibiting $\mathrm{CW}$ operation.

Here, we demonstrate in an all-fibered platform, MIR CW pumped parametric amplification in a tapered $\mathrm{Ge}_{10} \mathrm{As}_{22} \mathrm{Se}_{68}$ photonic crystal fiber (PCF). The PCF is engineered to blue shift the zero dispersion wavelength (ZDW) in the thulium emission band and features single mode operation over a large wavelength span, low propagation losses, and high nonlinearity [4]. We obtained signal amplification of $4.5 \mathrm{~dB}$ and idler conversion efficiency (CE) of $2 \mathrm{~dB}$ in a one meter-long fiber with only $125 \mathrm{~mW}$ from a telecom pumped Tm-doped fiber laser [5]. This result nominates Chalcogenide glass $(\mathrm{ChG}) \mathrm{PCF}$ as one of the most promising waveguide platform for advanced nonlinear applications in MIR.

\section{Experimental result and discussion}

The cross-section of the PCF is composed by three hexagonal concentric rings of air holes and was initially designed to feature a diameter to pitch ratio $(\rho)$ of 0.6 with a core diameter of $4 \mu \mathrm{m}$. These parameters allow the maximum blue shift of the ZDW, near 3 micron, within a single drawing process. To further shift the ZDW in the 2 micron range, we tapered the fiber to have a core diameter in the waist region of about $1.5 \mu \mathrm{m}$. An optical image of the cross-section of the two different regions of the fiber is shown in Fig. 1a, while a sketch of the fiber with its parameters is reported in Fig. 1b. The set-up consists in two custom made Tm-doped fiber lasers pumped with Lband erbium-doped fiber amplifiers (EDFAs). The pump wavelength is selected by using different fiber Bragg gratings (FBGs), featuring a constant linewidth of about $0.08 \mathrm{~nm}$, while the signal wavelength is tunable from 1950 $\mathrm{nm}$ to $2050 \mathrm{~nm}$.

(a)

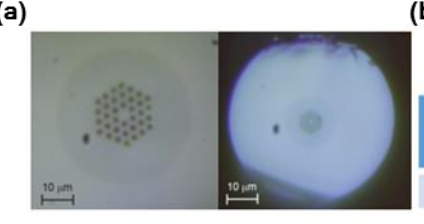

(b)

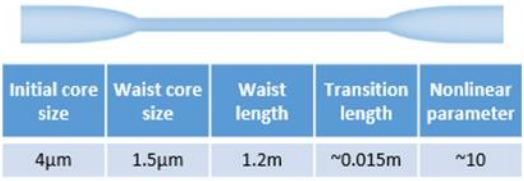

(c)

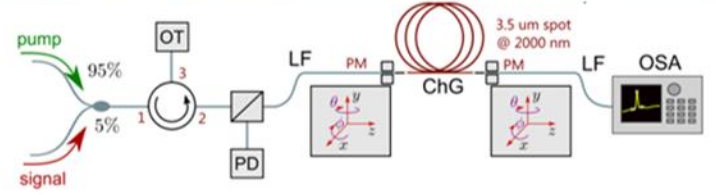

(d)

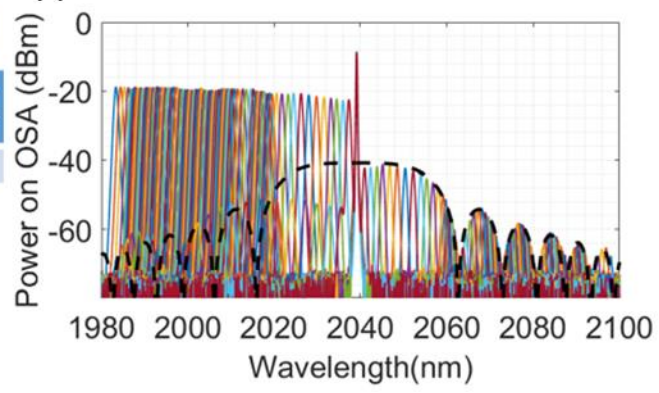

Figure 1: (a) Optical microscope image of the PCF un-tapered and tapered section. (b) Schematic of the whole fiber with a table resuming the main geometrical parameters. (c) Schematic of the experimental set-up. OT: optical terminator, PD: photodetector, LF: lensed fiber, OSA: optical spectrum analyzer. (d) FWM spectrum at the fiber output when $13 \mathrm{~mW}$ of pump power are coupled in the waist region.

The lasers are coupled and sent to the GeAsSe PCF through a fibered polarization beam splitter and a lensed fiber. A total insertion loss of $8.5 \mathrm{~dB}$ was measured at $1950 \mathrm{~nm}$, with approximately $4.5 \mathrm{~dB}$ of total coupling losses, $3.4 \mathrm{~dB}$ coming from the transition regions and $0.6 \mathrm{~dB}$ coming from propagation losses $(\alpha)$. A sketch of the experimental setup is shown in Fig. 1c. From low power nonlinear characterizations by four wave mixing (FWM) at different pump wavelengths, the nonlinear parameter $(\gamma)$ was estimated to be around $10 \mathrm{~W}^{-1} \mathrm{~m}^{-1}$, while the dispersion was 
found in agreement with simulations, where ZDW between 2.1 and $2.2 \mu \mathrm{m}$ is expected[6]. Fig. 1d shows an example where a pump wavelength of $2040 \mathrm{~nm}$ is employed. Clear phase matching features at the idler side confirm the quality and uniformity of the PCF.

Fig. 2a shows a clear ON/OFF signal amplification of almost $5 \mathrm{~dB}$ and cascaded FWM until $3^{\text {rd }}$ order when a pump power of $125 \mathrm{~mW}$ at $1950 \mathrm{~nm}$ is coupled in the waist region with a $1 \mathrm{~mW}$ signal. In addition, the linewidth of the converted waves well reproduces the signal one, thanks to the smaller linewidth of the pump laser. The CE was calculated as the idler power over the signal power at taper waist input. $\mathrm{CE}$ as a function of signal detuning was plotted at $125 \mathrm{~mW}$ coupled power level (Fig. 2b). The idler reaches transparency condition near the pump and has a $20 \mathrm{~dB}$ bandwidth of $15 \mathrm{~nm}$. ON/OFF signal amplification is reported in Fig. $2 \mathrm{c}$ as a function of signal wavelength for different pump power. We observe amplification for a coupled power as low as $65 \mathrm{~mW}$, and a reduction of the signal amplification bandwidth increasing the pump power. This agrees with theory as we are pumping in normal dispersion regime, and the nonlinear phase mismatch contribution reduces the frequency range over phase matching is achieved.
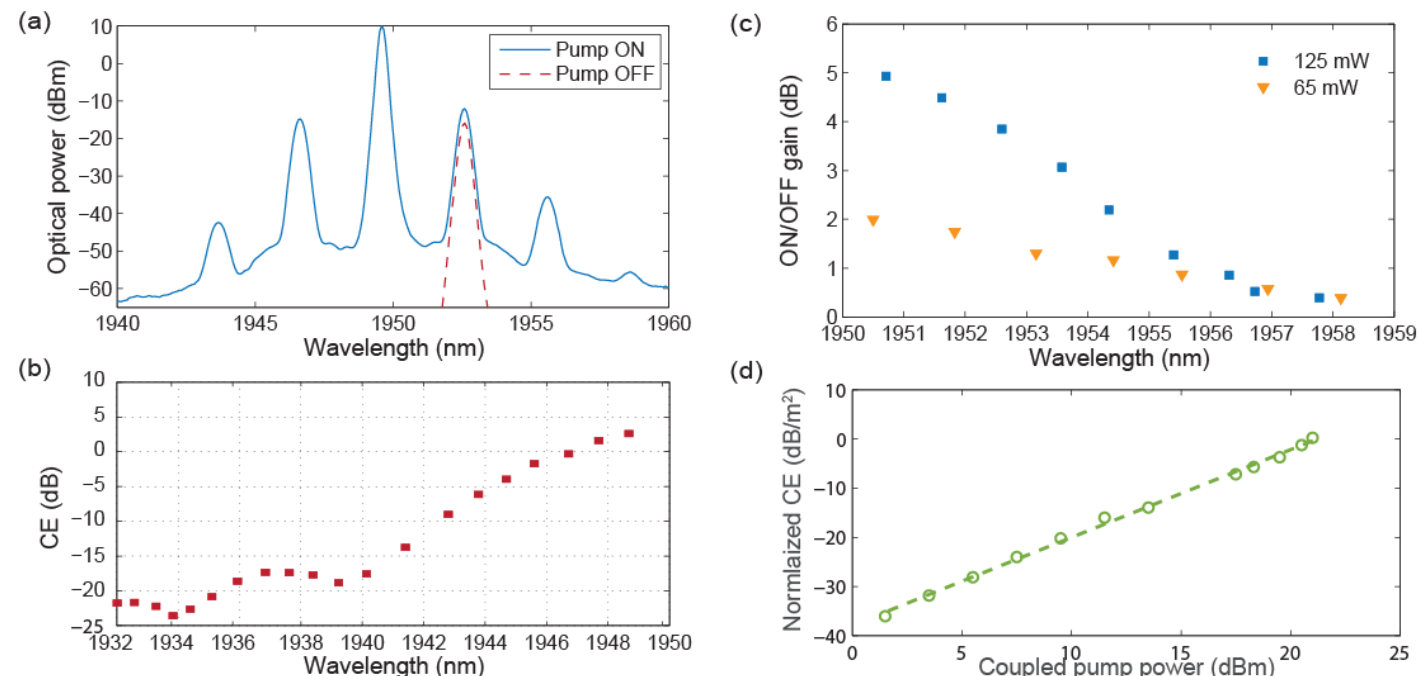

(d)

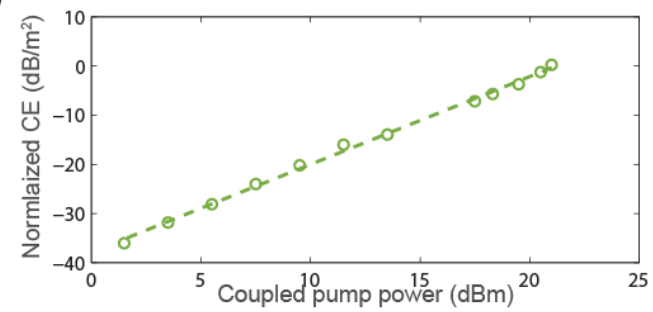

Figure 2: (a) The blue curve shows the output spectrum when $125 \mathrm{~mW}$ from the pump laser are coupled into the waist region. The dotted red curve represents the transmission of the signal when the pump is turned off. (b) Idler conversion efficiency as a function of the signal wavelength at $125 \mathrm{~mW}$ of coupled pump power. (c) ON/OFF signal amplification for different pump power. (d) CE as a function of coupled pump power.

The CE-pump power relation is shown in Fig 2d. No saturation is observed at high power, indicating no higher photon absorption process is present. The curve fitting shows a slope slightly less than 2 . The discrepancy might be due in part to the cascaded FWM, as observed in Fig. 2a. When the pump wave acts as a signal, it can be strong enough to deplete the signal and idler waves, acting now as the pump, in the generation of higher order side bands.

In summary, we achieved parametric amplification at $2 \mu \mathrm{m}$ using low power $\mathrm{CW}$ fiber lasers. This result was possible by careful dispersion engineering and state of the art fabrication techniques applied to a GeAsSe PCF. The fiber showed excellent power handling capabilities as intensity was estimated to be as high as $6.62 \mathrm{MW} / \mathrm{cm}^{2}$ in the waist region. Moreover, recently has been shown robust splicing between chalcogenide fibers with silica fibers [7], enabling compatibility with current fiber based devices and reducing coupling losses. Higher conversion efficiency and bandwidth are expected by pumping at the ZDW or slightly in the anomalous regime. This can be achieved both by further reducing the fiber core size or increasing its diameter-to-pitch ratio, and by employing thulium-holmium (Tm-Ho) co-doped fiber lasers [8], to red shift the pump emission wavelength.

This work was supported by the European Research Council under grant agreement ERC-2012- StG 306630-MATISSE

[1] J. Hansryd, et al., "Fiber-based optical parametric amplifiers and their applications," in IEEE Journal of Selected Topics in Quantum Electronics, 8, 506 (2002).

[2] X. Liu, et al., "Mid-infrared optical parametric amplifier using silicon nanophotonic waveguides," Nat. Photonics 4, 557-560 (2010).

[3] N. Abdukerim, L. Li, and M. Rochette, "Chalcogenide-Based Optical Parametric Oscillator," 25-26 (2016).

[4] S. Xing, et al., "Characterization and modeling of microstructured chalcogenide fibers for efficient mid-infrared wavelength conversion," Opt. Express 24, 9741 (2016).

[5] S. Kharitonov and C.-S. Brès, "Isolator-free unidirectional thulium-doped fiber laser," Light Sci. Appl. 4, e340 (2015).

[6] S. Xing, et al., "Small core Chalcogenide photonic crystal fiber for mid- infrared wavelength conversion : experiment and design," Cleo:2016, STh1O.6 (2016).

[7] R. Thapa, et al., "Low-loss, robust fusion splicing of silica to chalcogenide fiber for integrated mid-infrared laser technology development," Opt. Lett. 40, 5074 (2015).

[8] S. D. Jackson, “Towards high-power mid-infrared emission from a fibre laser,” Nat. Photonics 6, 423-431 (2012). 